\title{
Impact of the chemokine receptors CXCR4 and CXCR7 on metastatic potential and survival in adrenocortical carcinoma
}

Irina Chifu ${ }^{1}$, Carmina Fuß ${ }^{1}$, Cristina Ronchi ${ }^{1}$, Katja Marienfeld ${ }^{1}$, Martin Fassnacht ${ }^{1}$, Stefanie Hahner ${ }^{1}$, Britta Heinze ${ }^{1}$

${ }^{1}$ Department of Endocrinology and Diabetology, University Hospital Würzburg, Germany

INTRODUCTION Apart from their well-established physiological functions, chemokines appear to play a central role in tumor progression and metastasis in a large number of human cancers. Our study aims to evaluate the prognostic value of CXCR4 and CXCR7 expression in adrenocortical carcinoma (ACC) with regard to developing a new theranostic concept in this tumour entity.
ACC

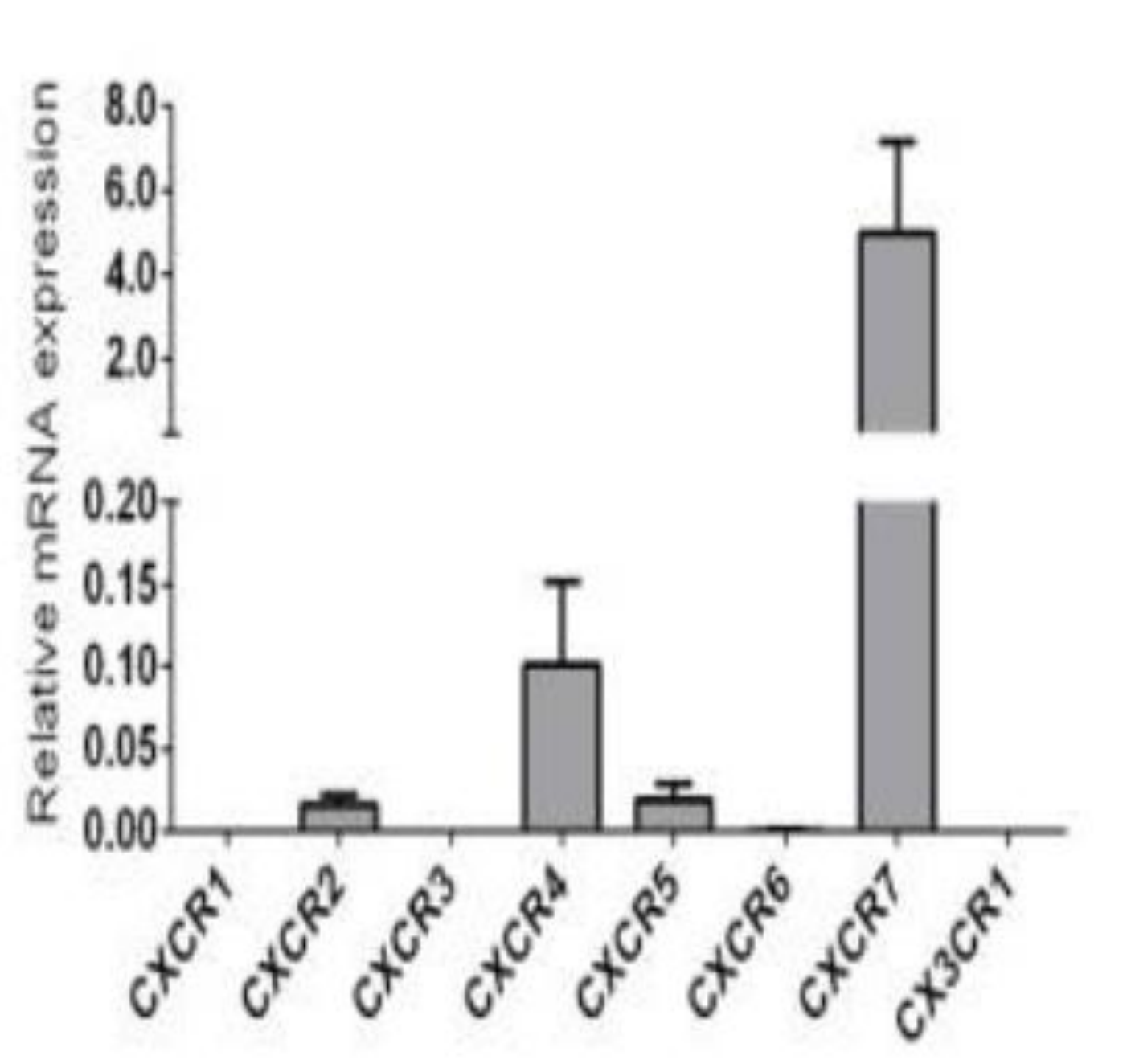

Adrenal gland

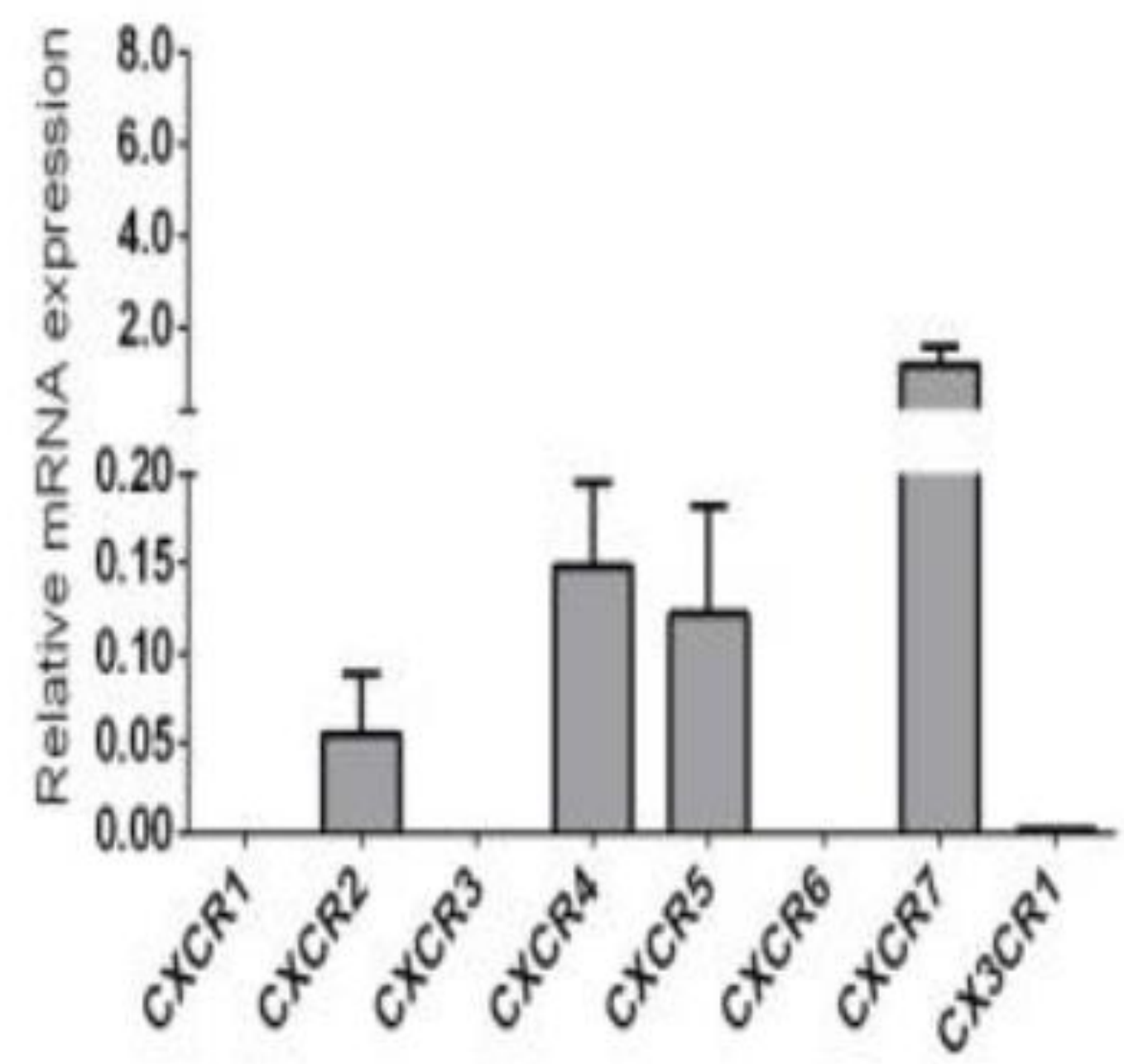

$\mathrm{NCl}-\mathrm{H} 295$ cells

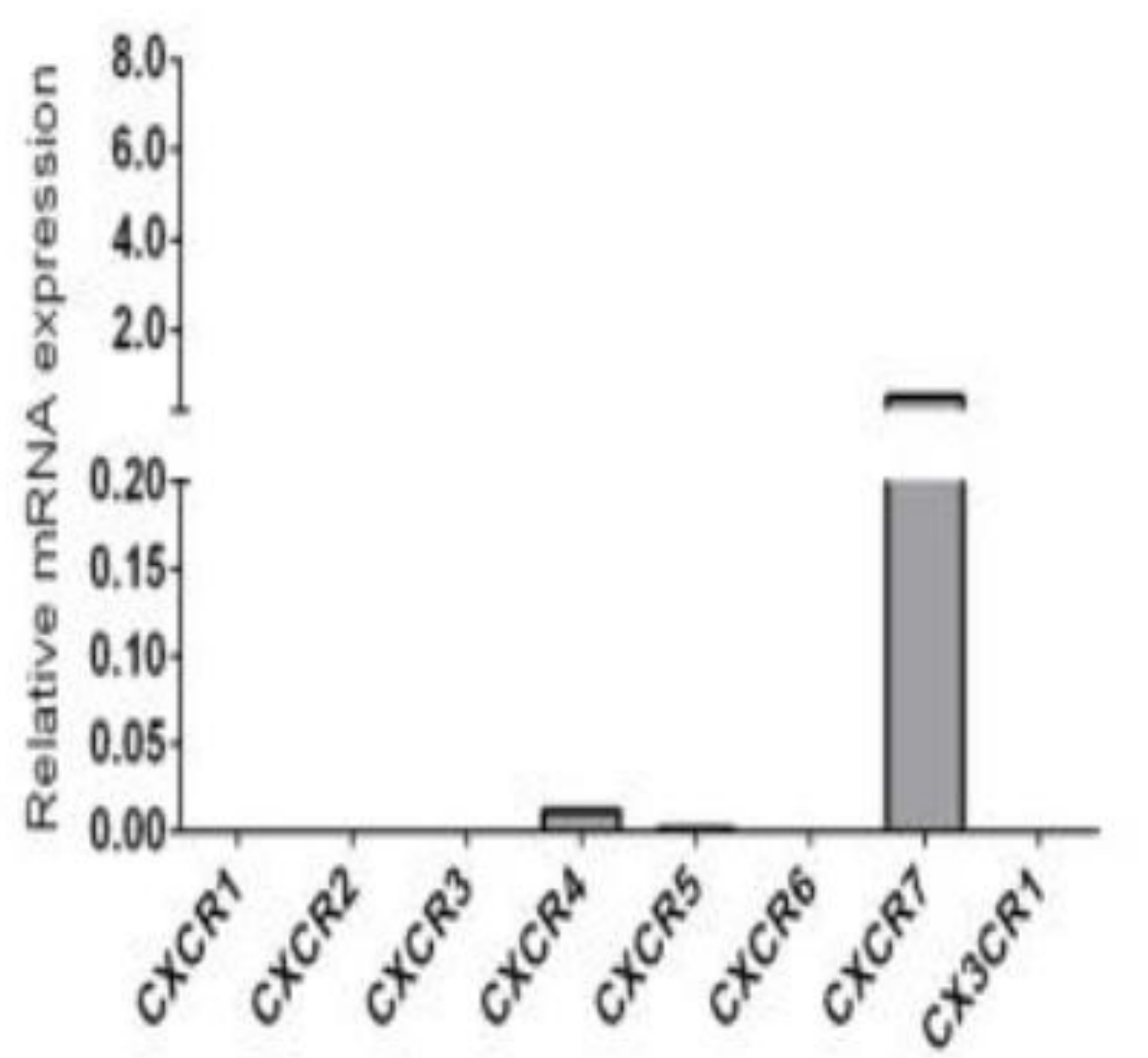

Fig.2 Real Time PCR. Quantitative analysis of chemokine receptor mRNA levels in 4 normal human adrena glands, 18 adrenocortical carcinomas and in the human adrenocortical carcinoma cell line $\mathrm{NCl}$-h295
METHODS Expression of seven CXC-chemokine receptors was assessed by quantitative real-time PCR in 4 normal adrenals, $18 \mathrm{ACC}$ and $\mathrm{NCl}-\mathrm{H} 295$ cells. CXCR4 and CXCR7 expression was further investigated by immunohistochemistry in paraffin-embedded sections of 215 ACC tissues (174 primary tumors (PT), 18 local recurrences (LR), 23 metastases (M)). 46.6\% $(n=104)$ of patients had an initial $\mathrm{RO}$ resection status. Data were correlated with metastatic status, tumor progress and survival.

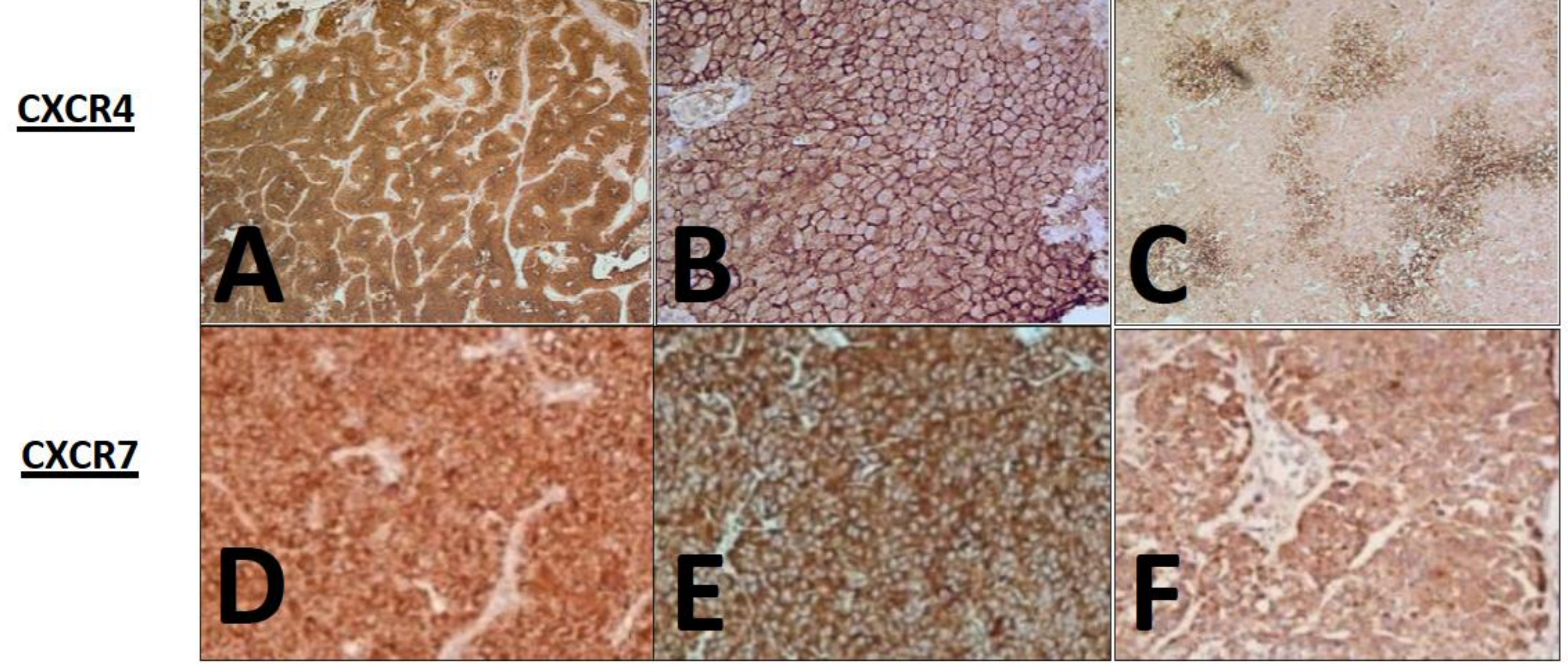

Fig.1 Immunohistochemical staining of CXCR4 (A-C) and CXCR7 (D-F) in adrenocortical carcinoma. A, D-E Example for strong membranous and cytoplasmic staining (magnification 10x, carcinoma. A, D-E Example for strong membranous and cyloplasmic staining (magnifation 10x, 20X). B Example for strong CXCR4 membrane staining (magnification10x). C Example for high membranous and cytoplasmic staining (magnification 20x)
Membrane

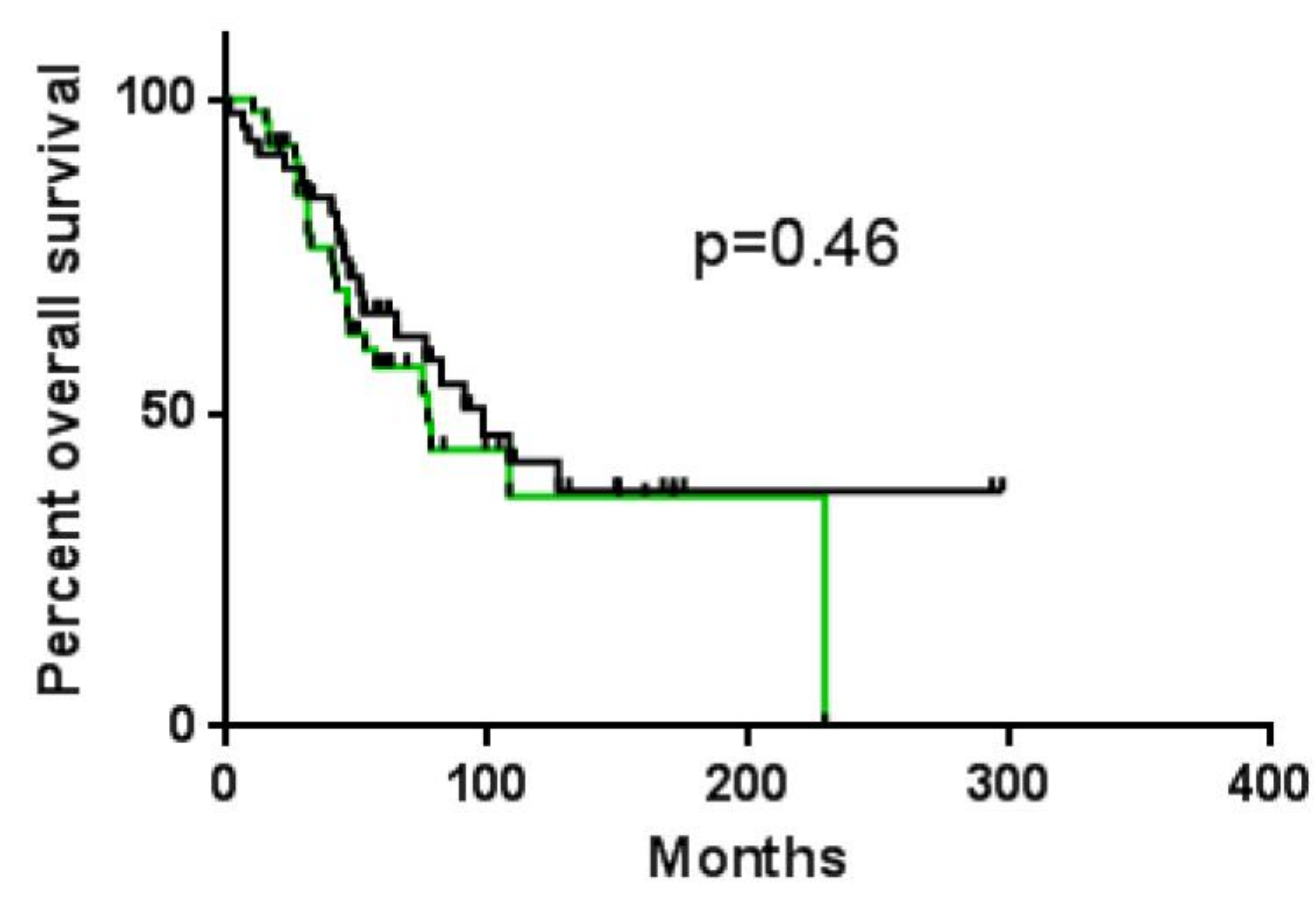

Cytoplasm

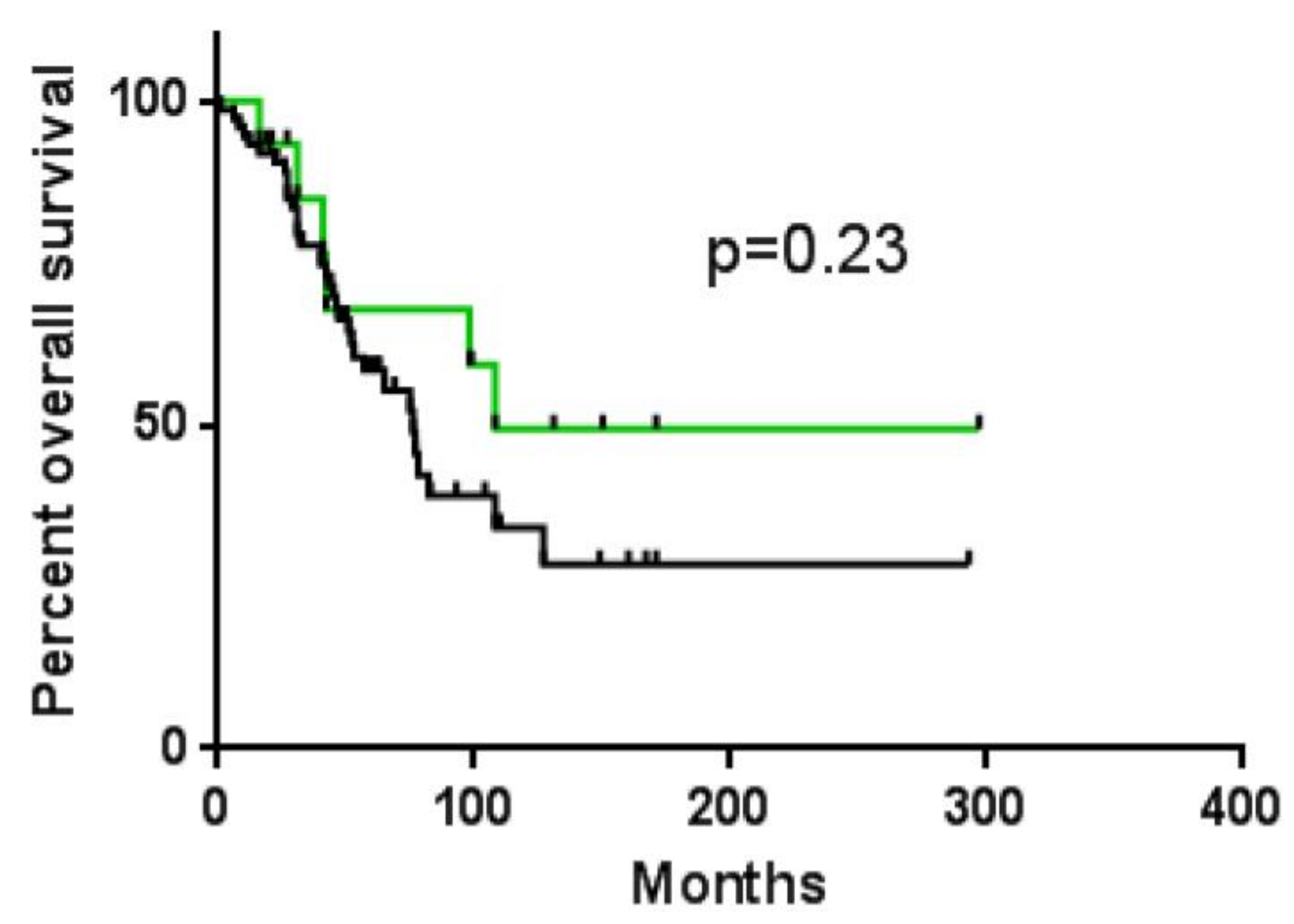

Fig.3 Correlation of overall survival of 102 R0-resected patients with CXCR4 membrane and cytoplasm expression; Kaplan Meier analysis

A

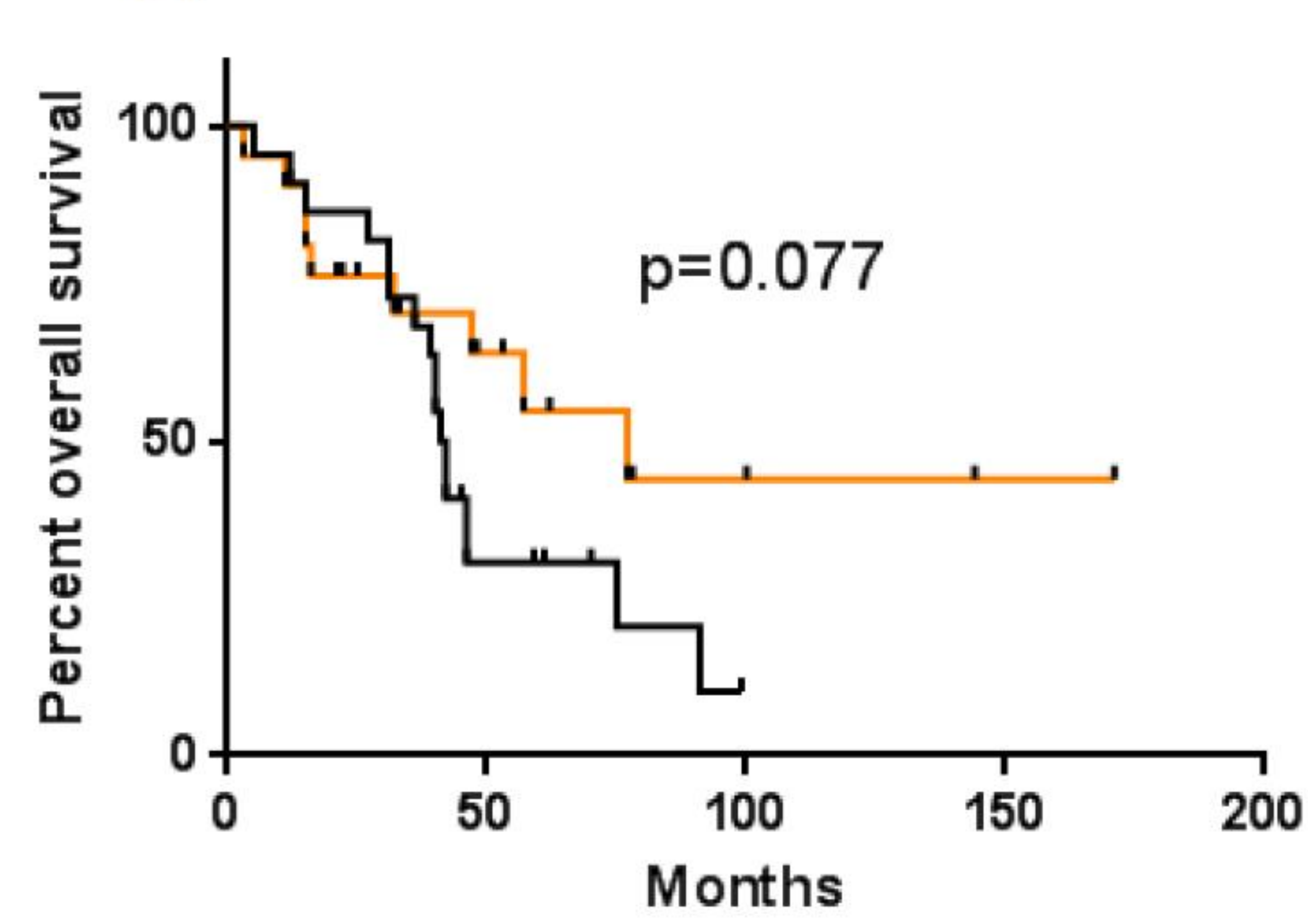

B

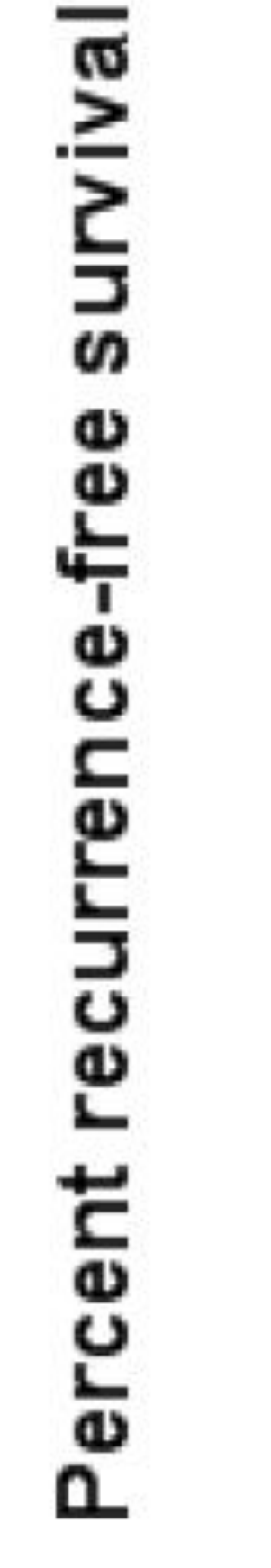

$+\mathrm{CXCR} 4 \mathrm{neg}(\mathrm{H}-\mathrm{score} \leq 1)$ + CXCR4 pos $(\mathrm{H}-\mathrm{score}>1)$

Fig.4 CXCR7 expression: correlation with outcome in patients with low ENSAT stage $(I+I I)$ : A Impact of CXCR7 membrane expression on overall survival ( $n=43$ ) $B$ Impact of CXCR7cytoplasm expression on recurence-free survival $(n=11)$

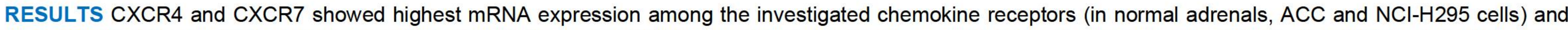

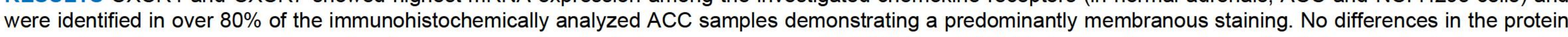

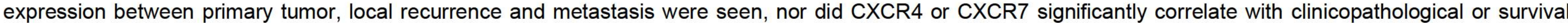

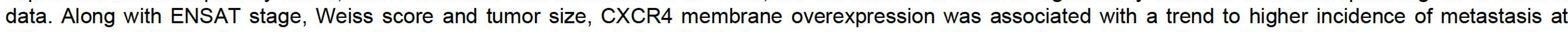

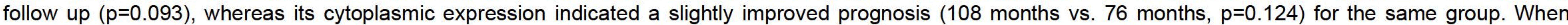

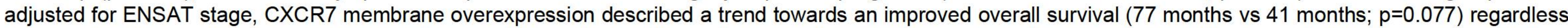

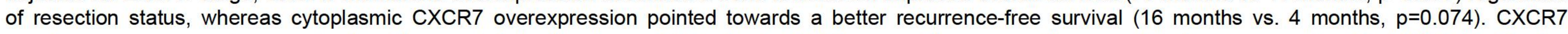

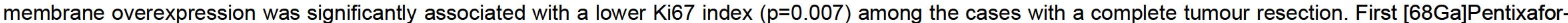

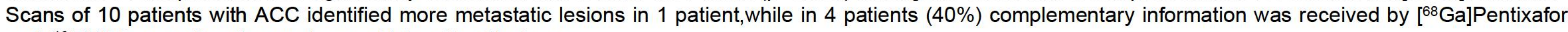
and $\left[{ }^{18} \mathrm{~F}\right] \mathrm{FDG}$ regarding the number and intensity of lesions.

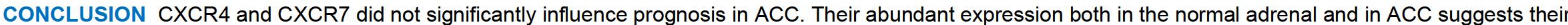

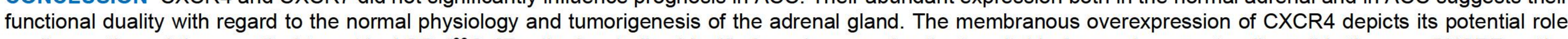

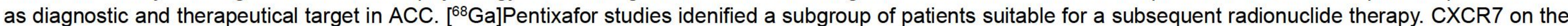
other hand appears to be a marker of a less aggressive tumor phenotype. 